PROCEEDINGS OF THE AMERICAN MATHEMATICAL SOCIETY

Volume 126, Number 5, May 1998, Pages 1311-1314

S 0002-9939(98)04607-3

\title{
THE RIEMANN HYPOTHESIS, SIMPLE ZEROS AND THE ASYMPTOTIC CONVERGENCE DEGREE OF IMPROPER RIEMANN SUMS
}

\author{
JONATHAN SONDOW \\ (Communicated by Dennis A. Hejhal)
}

\begin{abstract}
We characterize the nonreal zeros of the Riemann zeta function and their multiplicities, using the "asymptotic convergence degree" of "improper Riemann sums" for elementary improper integrals. The Riemann Hypothesis and the conjecture that all the zeros are simple then have elementary formulations.
\end{abstract}

\section{Outline}

In $\S 2$, as a measure of how fast a sequence converges or diverges, we define its "asymptotic convergence degree". This is applied in $\S 3$ to sequences of "improper Riemann sums" for elementary improper integrals, yielding real-valued functions of a complex variable. Their points of discontinuity are used in $\S 4$ to characterize the nonreal zeros of $\zeta(s)$ and their multiplicities. The Riemann Hypothesis and the conjecture that all the zeros are simple are then formulated in elementary terms (Corollaries 2 and 3 ). In $\S 5$ we compute some examples, and $\S 6$ contains the main proof.

In a paper in preparation, the author will extend these results to more general (improper) Riemann sums and integrals.

\section{The Asymptotic CONVERGEnCE DEGREe}

Let $\left\langle S_{N}\right\rangle=\left\{S_{1}, S_{2}, S_{3}, \ldots\right\}$ denote an infinite sequence of complex numbers. For a complex number $I$, we say $S_{N}-I=O\left(N^{-a}\right)$ if there exists a constant $c>0$ such that $\left|S_{N}-I\right|<c N^{-a}$ for $N \rightarrow \infty$. Define the asymptotic convergence degree $\alpha\left(\left\langle S_{N}\right\rangle, I\right)$ to be the element of the extended real number system $[-\infty, \infty]$ given by

$$
\alpha\left(\left\langle S_{N}\right\rangle, I\right)=\sup \left\{a: S_{N}-I=O\left(N^{-a}\right)\right\}
$$

if the set of all such $a$ is nonempty; otherwise $\alpha\left(\left\langle S_{N}\right\rangle, I\right)=-\infty$.

For example, $\alpha\left(\left\langle N^{-s} \log N\right\rangle, 0\right)=\operatorname{Re}(s)$, but $\alpha\left(\left\langle N^{-1}\right\rangle, 1\right)=0$.

Evidently, if $\alpha=\alpha\left(\left\langle S_{N}\right\rangle, I\right)>0$, the sequence converges to $I$, while if $\alpha<0$, the sequence is unbounded.

Received by the editors October 28, 1996.

1991 Mathematics Subject Classification. Primary 11M26; Secondary 40A05.

(C)1998 American Mathematical Society 


\section{IMPROPER RIEMANN SUMS}

For $s \in \mathbf{C}$ and $N \geq 1$, the averages

$$
S_{N}(s)=\frac{1}{N} \sum_{n=1}^{N} \frac{1}{(n / N)^{s}}
$$

are Riemann sums for the integral

$$
\int_{0}^{1} \frac{1}{x^{s}} d x
$$

when $\sigma:=\operatorname{Re}(s) \leq 0$. If $\sigma>0$, the integral is improper and we call $S_{N}(s)$ an improper Riemann sum.

On the half-plane $\sigma<1$, the integral defines a function whose analytic continuation over the punctured plane $\mathbf{C}-\{1\}$ is

$$
I(s)=\frac{1}{1-s} .
$$

Taking $m \geq 0$ derivatives of $S_{N}(s)$ and $I(s)$, we set

$$
\alpha_{m}(s)=\alpha\left(\left\langle S_{N}^{(m)}(s)\right\rangle, I^{(m)}(s)\right)
$$

so that, for instance, $\alpha_{0}(0)=\alpha\left(\left\langle S_{N}(0)\right\rangle, I(0)\right)=\infty$.

\section{Zeros of THE ZeTA FUNCTION}

Theorem 1. (i) Except that $\alpha_{0}(0)=\infty$, the asymptotic convergence degree $\alpha_{m}(s)$ is finite for all $s \neq 1, m \geq 0$, and is positive, negative or zero according as $\sigma$ is $<1,>1$ or $=1$, respectively.

(ii) The function $\alpha_{0}(s)$ is continuous on $\mathbf{C}-\{0,1\}$ except for a jump of $+\operatorname{Re}(\rho)$ at each nonreal zero $\rho$ of the Riemann zeta function.

(iii) For $m \geq 1$, the function $\alpha_{m}(s)$ is continuous on $\mathbf{C}-\{1\}$ except for jumps of $+(\operatorname{Re}(\rho)+1+2\lfloor m / 2\rfloor)$ at zeta zeros of multiplicity $>m$ (if any).

Here $\lfloor x\rfloor$ denotes the integer in the half-open interval $(x-1, x]$.

The proof of Theorem 1 is given in $\S 6$.

For $\sigma>0$, we see from (i) or, for $\sigma=1$, from (3) in $\S 6$, that the sequence of improper Riemann sums $\left\langle S_{N}(s)\right\rangle$ and the improper integral (1) both converge $(\sigma<1)$ or both diverge $(\sigma \geq 1)$; similarly for the $m$ th derivatives.

In view of the functional equation, (ii) implies

Corollary 2. The Riemann Hypothesis is equivalent to the function

$$
\alpha_{0}(s)=\sup \left\{a: \frac{1}{N} \sum_{n=1}^{N} \frac{1}{(n / N)^{s}}=\int_{0}^{1} \frac{1}{x^{s}} d x+O\left(\frac{1}{N^{a}}\right)\right\}
$$

being continuous on either the strip $0<\sigma<1 / 2$ or $1 / 2<\sigma<1$.

By (iii), for $m>0$ the logarithm factors implicit in the definition of $\alpha_{m}(s)$ somehow smooth out the jumps that $\alpha_{0}(s)$ has at nonreal zeros of multiplicity $\leq m$. The trivial zeros being simple, we have

Corollary 3. The function

$$
\alpha_{1}(s)=\sup \left\{a: \frac{1}{N} \sum_{n=1}^{N} \frac{\log (n / N)}{(n / N)^{s}}=\int_{0}^{1} \frac{\log x}{x^{s}} d x+O\left(\frac{1}{N^{a}}\right)\right\}
$$


is continuous on the open critical strip if and only if $\zeta(s)$ has only simple zeros. In general, for $m>0$ the function $\alpha_{m}(s)$ is continuous on the strip $0<\sigma<1$ if and only if $\zeta(s)$ has no zeros of multiplicity $>m$.

\section{EXAMPLES}

Formula (4) in $\S 6$ includes the remarkable fact that the asymptotic convergence degree $\alpha_{0}(s)$ jumps from $1-\sigma$ to 1 at a complex zeta zero. (Indeed, by (3), for $\sigma=1 / 2$ the bounds

$$
\frac{1}{N} \sum_{n=1}^{N} \frac{1}{(n / N)^{s}}-\int_{0}^{1} \frac{d x}{x^{s}}= \begin{cases}O\left(\frac{1}{\sqrt{N}}\right) & \text { if } \zeta(s) \neq 0, \\ O\left(\frac{1}{N}\right) & \text { if } \zeta(s)=0\end{cases}
$$

are best possible.) To illustrate this phenomenon, we compute $\left|S_{N}(s)-I(s)\right|$ (truncated) at a point $1 / 2+13 i$ where $\zeta(s) \neq 0$, and at the first zero $\rho_{1}=$ $1 / 2+(14.13472 \ldots) i$, for three values of $N$ :

\begin{tabular}{|c|ccc|}
\hline & \multicolumn{3}{|c|}{$\left|S_{N}(s)-I(s)\right|$} \\
\hline$s$ & $N=10$ & $N=100$ & $N=1000$ \\
\hline $1 / 2+13 i$ & .22243 & .07563 & .02534 \\
$\rho_{1}$ & .05091 & .00499 & .00049 \\
\hline
\end{tabular}

\section{Proof of The TheOREM}

Put $K=1+\left\lfloor\frac{1}{2} \max (m,-\sigma)\right\rfloor$. Then $\sigma>-2 K$, so the Euler-Maclaurin summation formula [1, pp. 114-115] gives the analytic continuation

$$
\zeta(s)=\sum_{n=1}^{N} \frac{1}{n^{s}}+\frac{N^{1-s}}{s-1}-\frac{1}{2 N^{s}}+\sum_{j=1}^{K} \frac{(s)_{2 j-1} B_{2 j}}{(2 j) ! N^{s+2 j-1}}+R_{2 K}
$$

for $N \geq 1$, where the $B_{2 j}$ are (nonzero) Bernoulli numbers, $(s)_{j}$ denotes the product $s(s+1) \cdots(s+j-1)$, and the error term is

$$
R_{2 K}=R_{2 K}(s, N)=\frac{(s)_{2 K+1}}{(2 K+1) !} \int_{N}^{\infty} \frac{\bar{B}_{2 K+1}(x)}{x^{s+2 K+1}} d x
$$

Here $\bar{B}_{2 K+1}(x)$, a "periodified" Bernoulli polynomial, is bounded.

Now divide (2) by $N^{1-s}$ and write the result as

$$
\frac{1}{N} \sum_{n=1}^{N} \frac{1}{(n / N)^{s}}-\frac{1}{1-s}=\frac{\zeta(s)}{N^{1-s}}+\frac{1}{2 N}-\sum_{j=1}^{K} \frac{(s)_{2 j-1} B_{2 j}}{(2 j) ! N^{2 j}}-\frac{R_{2 K}}{N^{1-s}} .
$$

The last term being $O\left(N^{-2 K-1}\right)$, we deduce for $s \neq 1$ that

$$
\alpha_{0}(s)= \begin{cases}1 & \text { if } \zeta(s)=0 \\ \infty & \text { if } s=0, \\ \min (1,1-\sigma) & \text { otherwise }\end{cases}
$$

Next, for $m \geq 1$ and $s \neq 1$ we show that

$$
\alpha_{m}(s)= \begin{cases}2+2\lfloor m / 2\rfloor & \text { if } \zeta(s)=\zeta^{\prime}(s)=\cdots=\zeta^{(m)}(s)=0, \\ \min (2+2\lfloor m / 2\rfloor, 1-\sigma) & \text { otherwise. }\end{cases}
$$


To see this, annihilate the terms with $j<k:=1+\lfloor m / 2\rfloor$ in the summation on the right side of (3) by differentiating the equation $m$ times. Estimating the error terms and using $k \leq K$, we obtain

$$
\begin{aligned}
S_{N}^{(m)}(s)-I^{(m)}(s)= & \sum_{j=0}^{m}\left(\begin{array}{c}
m \\
j
\end{array}\right) \zeta^{(j)}(s) \frac{(\log N)^{m-j}}{N^{1-s}} \\
& -\frac{D^{m}(s)_{2 k-1} B_{2 k}}{(2 k) ! N^{2 k}}+O\left(\frac{(\log N)^{m}}{N^{2 k+1}}\right)
\end{aligned}
$$

where $D=d / d s$. Note that

$$
\frac{D^{m}(s)_{2 k-1}}{(2 k-1) !}= \begin{cases}s+k-1 & \text { if } m=2 k-2 \\ 1 & \text { if } m=2 k-1\end{cases}
$$

This is nonvanishing if $s$ is a zero of multiplicity $>m \geq 1$, for then $s$ is not real; the first case of (5) follows. In the other case, (7) may or may not vanish. If it does, then $1-s=k<2 k$, which implies (5). If (7) does not vanish, then (5) holds, unless the $N^{2 k}$ term in (6) cancels the $N^{1-s}$ term with no log factor and the other $N^{1-s}$ terms vanish. But then $2 k=1-s$ and $\zeta(s)=0$, a contradiction. This completes the proof of (5).

Finally, since all complex zeta zeros lie in the strip $0<\sigma<1$, formulas (4) and (5) imply the theorem.

\section{REFERENCE}

1. H. M. Edwards, Riemann's Zeta Function, Academic Press, New York, 1974. MR 57:5922

209 West 97th Street, New York, New York 10025 